\title{
Revolutions in the earth sciences
}

\author{
Glaude Allègre ${ }^{1}$ and Vincent Gourtillot ${ }^{2}$ \\ ${ }^{1}$ French Minister of National Education, Research and Technology, ${ }^{2}$ Director of Research at the French Ministry of National Education, \\ Institut de Physique du Globe de Paris, 4 place Jussieu, 75005 Paris Cedex 05, France, and Université Denis Diderot Paris 7
}

\begin{abstract}
The 20th century has been a century of scientific revolutions for many disciplines: quantum mechanics in physics, the atomic approach in chemistry, the nonlinear revolution in mathematics, the introduction of statistical physics. The major breakthroughs in these disciplines had all occurred by about 1930. In contrast, the revolutions in the so-called natural sciences, that is in the earth sciences and in biology, waited until the last half of the century. These revolutions were indeed late, but they were no less deep and drastic, and they occurred quite suddenly. Actually, one can say that not one but three revolutions occurred in the earth sciences: in plate tectonics, planetology and the environment. They occurred essentially independently from each other, but as time passed, their effects developed, amplified and started interacting. These effects continue strongly to this day.
\end{abstract}

Keywords: history of science; revolutions; earth sciences; plate tectonics; planetology; environment

\section{PLATE TECTONICS}

Plate tectonics occurred primarily as a consequence of exploration of the oceans. The breakthrough is associated with the publication of the model built by P. Morley, F. Vine and D. Matthews to interpret the symmetry of magnetic anomalies on the ocean floor. The core of the theory is based on the existence of a few rigid plates, $70-100 \mathrm{~km}$ in thickness, all moving with respect to each other at the surface of the earth. They imply two crucial processes: surface creation by volcanism at ridge crests, and surface destruction or removal by collisions at mountain ranges or subduction at oceanic troughs.

Plate movements obey simple theorems of geometry on the sphere, as demonstrated by early mathematicians such as Leonhard Euler. The first-order model introduced a very new concept: that the main kinematic differences were not, as originally believed, between continent and ocean, but between one plate and another. Plate boundaries could go through continent to ocean boundaries. However, plates are heterogeneous and do contain special inclusions: the continents. These light pieces of crust move with the plates they belong to but cannot be reinjected directly into the mantle because of their low density. Because they remain at the surface of the earth, they have recorded, sometimes over a very long span of geological time, the different episodes of the earth's history.

Indeed, what makes the earth sciences so special among the so-called 'hard sciences' is that they share with some of the 'humanities' a fundamental dimensionthat of time, and more precisely that of 'long time', as introduced in history, for instance, by $\mathrm{H}$. Braudel. It is the ability to measure time, to go from a static to a kinematic and then to a dynamic picture of the earth over time-scales ranging from thousands to billions of years, that give the earth sciences much of their attraction for students, and much of their power. The earth sciences are historical sciences in that sense.
Among the most important episodes in the earth's history are those where continents have collided with each other and subsequently created high mountain ranges, such as the Alps or the Himalayas (to take some of the more recent examples), or those when a subduction zone has been initiated and started to generate an elongate range, such as the Andes, at the edge of a continent. Among the most important observations accounted for by the plate tectonic paradigm are the geography of earthquakes and volcanoes. Basically, most occur at present (or former) plate boundaries, where much of the energy flux coming from the interior of the earth is dissipated.

In many ways, plate tectonics may seem only to be a revival of the continental drift model developed by German meteorologist Alfred Wegener in the early part of the 20th century. But this is not quite the case: plate tectonics are far richer and have far stronger theoretical bases. The paradigm allows a very broad-ranging frame to be constructed, thanks to which many previously unrelated geological phenomena can be understood. As a consequence, many subdisciplines of the earth sciences, which previously had very little productive interaction, such as palaeontology, geochemistry, geomagnetism, seismology, tectonics, volcanology and stratigraphy, are now organized around a common subject of interest with predictive power. The earth is finally the true common subject of interest for all earth scientists.

Yet, what may be the most important component of plate tectonics is the fact that plate movements imply large-scale convective motion within the earth's mantle. The mechanical properties of materials in the mantle depend on the time-scale over which forces are exerted. At the short time-scale familiar to us, the earth's mantle is solid and rings like a bell when a large earthquake occurs. But over the much longer time-scales involved in geology, the mantle can flow like a viscous or plastic body. One could almost say that over such time-scales, mantle flow is not unlike that of water in a boiling pot. 
The energy sources that feed these convective motions include the original thermal and gravitational energies stored during the accretion and differentiation of our planet in its core, and also that generated by the radioactivity of long-lived radioactive isotopes of potassium, uranium and thorium $\left({ }^{40} \mathrm{~K},{ }^{238} \mathrm{U},{ }^{235} \mathrm{U}\right.$ and $\left.{ }^{232} \mathrm{Th}\right)$.

Observational constraints on the mechanics of convection in the earth's mantle, that is on the engine driving plate tectonics, come mainly from two different methods: seismic tomography and isotope geochemistry. Seismic tomography is the three-dimensional imaging of the earth's interior, using earthquakes as the source and observing seismic waves at the surface using the worldwide network of seismic receptors, processed with a set of methods akin to ultrasonic tomography in medicine. (Note that seismic tomographic methods were in fact the basis for medical tomography, which has become more popular for obvious reasons.) Isotope geochemistry uses isotopic ratios, which vary as a consequence of differential radioactive decay of several long-lived radioactive isotopes. They provide tracers of the earth's internal movements.

Seismic tomography gives a present-day image of mantle structure. It has made tremendous progress over the past few years, and improved resolution now allows one to follow the fate of subducted plate slabs as they sink to the so-called transition zone, some $670 \mathrm{~km}$ deep in the mantle, or as they cross what was until recently thought by many to be an impassable boundary and sink to the base of the mantle, accumulating in so-called 'slab graveyards'. A complementary circulation is also beginning to be imaged in the form of large, buoyant, uprising plumes, though only the largest ones are beginning to be seen.

Geochemistry gives an integrated historical image of convection. It bears the signature of time. The two techniques are therefore complementary.

We are now reaching a stage where reconstruction of past plate kinematics, based primarily on geophysical observations of the ocean floor (magnetic stripes and orientation of the so-called transform faults), can be blended into this picture to assign specific mantle anomalies, identified as subducted slabs in the tomographic models, to a given age and plate boundary, going back several hundred million years.

Observations can be blended into models thanks to remarkable advances in the ability to construct complex and accurate numerical models of the earth's convective motions under a variety of assumptions. These calculations outline the potential importance of large changes in viscosity, turbulent, chaotic nonlinear flow, and slab avalanches or plume eruptions. One cannot do without these numerical tools, though of course they should always be considered as tools and analysed harshly under the light of observational constraints. One should be wary of the danger of mistaking the beautiful images coming from these computations with reality.

\section{PLANETARY SCIENCES}

The revolution in the planetary sciences began with the first lunar exploration programmes: Apollo in the USA and Luna in the former USSR. The exploration of Mars, Mercury, Venus and then the giant planets of the outer solar system soon followed. We recall the awe and excitement as the Vyager and Galileo missions returned extraordinary pictures of planets, satellites and planetary rings. The diversity, the nature and history of other planetary objects was making it clear that the earth was but a member of a family, which could now be studied and modelled. The ubiquity of ring structures - sometimes fragmented - the latest discovered around Neptune and named after Liberté, Egalité, Fraternité and Courage; the ubiquity of volcanism in many varied forms from the colourful ongoing sulphur eruptions on Io, to the water and ammonia ice flows of Europe, Ganymede or Encelade; the joint presence of asteroid impacts and tectonic cracks, some due to the powerful tides of the main planet acting on the crusts of its tiny satellites. The images brought back are not restricted to photographs, but also provide observations and measurements of a number of physical parameters. Think of the truly remarkable recent radar images or detailed gravity maps of Venus obtained by the Magellan spacecraft: the spectacular topography, volcanic activity and tectonics of our sister planet revealed despite the thickness and opacity of its cloud systems.

Every single planet whose hard surface could be observed, either directly or remotely under cloud cover, revealed the presence of impact craters. Detailed mapping of these craters and direct age determinations of samples brought back from the moon allowed a strong relationship between crater density and surface age to be established. The first billion years of the solar system was a time of heavy bombardment, which decreased drastically thereafter. It was soon realized that it was because of plate tectonics and erosion that the earth's surface was so youthful and poor in impact craters. But because the moon lacked such active dynamics (both internal and external), it allowed us to read the early history of planetary formation.

As a result, the study of the early earth and solar system was completely revived and took new importance and significance. Meteorite impacts and crater formation must have been a key geological factor in this early earth. Isotope geochemistry was revealing details of this early history, going back almost 4 Gyr on the oldest surviving continental nuclei, and even before that on the moon, all the way back to $4.5 \mathrm{Gyr}$ ago. It was realized that meteorites are the most primitive objects in the system, both in age and composition: they are invaluable witnesses of the dawn of its history.

Cosmochemistry, a branch of isotope geochemistry, allowed the discovery of extinct radioactivities which complemented information carried by long-lived radioactive elements: the various events of solar system formation could be arranged in a solid reference frame with ultraprecise dating of each event. We now know for instance that early condensation of planetary material (planetesimals) occurred 4567 million years (Myr) ago, that earth was essentially completed and differentiated, with its metallic core and primitive outgassed atmosphere, 4400 Myr ago. Between these two dates, planetary objects were formed, broken, accreted again, going from dust to pebble, from smaller to larger ball size, from planetesimals to planets. The moon was, in a way, blown out as a result of one of several huge impacts suffered by the 
growing earth. The earth had not completed its differentiation when material was ejected by this impact.

The discovery of isotopic anomalies in primitive meteorites has allowed a full link between astronomers and geologists, through the joint techniques of cosmochemistry and geochemistry. The scenario of planetary formation involves nucleosynthesis and has one or several supernovae exploding less than 1 Myr before formation of the solar system and acting as the trigger. We now see the 12 or so Gyr since the occurrence of the Big Bang as a multitude of hypernovae formations, evolutions and explosions spawning formation of other solar systems. A unified scenario emerges but of course research goes on.

\section{ENVIRONMENTAL SCIENCES}

Pioneers such as Clair Patterson, who became concerned by lead poisoning that could be traced to fuel consumption, or Roger Revelle, who began to see the influence man could have on world climate, were long ignored by the general public as well as by many of their fellow scientists.

But the effects increased, their reality became inescapable, awareness and anxiety grew in the Western world and such topics became political issues. This was the rise of environmental sciences, sometimes improperly labelled as ecology. Recent issues include global warming, the ozone hole and the loss of biological diversity.

The loss of ozone in the high atmosphere was first identified over Antarctica and has now been confirmed there and found also above the Arctic. Its non-uniform but growing amplitude is now well documented, although the detailed mechanics, popularized through the scavenging role of chlorine components released by refrigerating systems mainly in the Northern Hemisphere, are still a topic of active research.

Of course the most popular notion is that of global warming. The regular increase in carbon dioxide content of the atmosphere was documented in the preserved environment of the Hawaiian volcanoes, and global warming was attributed to the uncontrolled release in the atmosphere of enormous amounts of $\mathrm{CO}_{2}$ linked to burning of coal and fuel, and deforestation by humans. Increasing greenhouse gases led to an overall rise in the mean temperature of the lower atmosphere, with potentially disastrous effects on population survival.

There is no doubt that man is the first species to become a geological agent of global significance. We now move every year more dirt, soil and earth than is brought to the world oceans by all large rivers through natural erosion and transport. However, the direct link between $\mathrm{CO}_{2}$ and temperature, and the exact nature of the causal mechanisms, remain topics of research. Clearly, our history and the length of time over which we have measurements is short by geological standards, and we do not yet know fully the time-scales and amplitudes of natural atmospheric and climatic changes. The memory of past climates is recorded in the tiny bubbles trapped in the ice retrieved in Arctic and Antarctic cores. Climate changes are modulated through the famous Milankovic cycles, due to changes in solar insulation, linked with changes in parameters of the earth's orbit around the sun. In that case, it is these planetary motions that drive insu- lation, then atmosphere and ocean temperatures, and finally $\mathrm{CO}_{2}$ concentration in the atmosphere, and not the reverse.

But it is clear that we are undergoing climate change on the scale of decades, and at the same time altering some of our cultural patterns in ways that may increase the variability of climatic extremes and the way they affect us. We should talk of increased climate perturbation possibly as much as of global warming. Awareness of these problems has generated intense scientific activity and international programmes to try to understand the interaction between oceans and atmosphere. A historical, then geological record of climate is slowly being built. Again, awareness and measurement of 'deep time', understanding of embedded time-scales, occurrence of nonlinear phenomena at several widely different space scales (such as the now famous and irregular El Niño) are essential ingredients of scientific advance.

Many other related or unrelated environmental events remain to be studied: the water cycle and evolution of water reserves (many say that water, not oil, will be the source of conflicts in the coming century), acid rain, sea and ocean pollution, ecological equilibrium, loss of biodiversity, floods, management of waste. All of these important problems may have solutions but require full realization of the deep unity and inter-connectedness of planetary subsystems, and of the planet as a whole: man may be saved or lost with it, although clearly man is not all of nature, which could well move forward after his disappearance.

\section{AND NOW?}

The three revolutions in the geosciences - the birth of plate tectonics, planetary sciences and environmental sciences-have some differences, but they share many common features. Moreover, having come close to each other in time, they have interacted strongly, and evolution of understanding in one has strongly and positively affected the others, possibly leading to a global paradigm at the highest scale.

As is the case for many scientific revolutions, these scientific and historical turning points appear to have surfaced as random events, based on chance observations. They were largely unpredicted and may have been unpredictable, but are now inescapable. The development of plate tectonics was largely a result of ocean exploration. Much of the initial motivation was on developing weapons for submarine warfare in the Second World War. The United States' Navy and continued generous funding of ocean exploration after the war, eventually led to the key discoveries and observations on which the new paradigm could be based. Planetary exploration was a byproduct of the Cold War, of the startling launching of the first Sputnik and the fight-back spirit that led John Kennedy to pledge landing on the moon within a decade. Environmental sciences were also the result of continuous technological progress, but stemmed from reaction of a certain generation in the Western world to perceived loss in the quality of life, with both a real basis and also some aspirations to a 'lost natural paradise'. The rise of the Green parties was a direct consequence of this perception. 
Of course all three revolutions were next fed by the genuine desire for a better understanding of nature, which drives scientific research. But they have evolved (in varying degrees) away from the purely descriptive status of the 19th-century natural sciences. They risk remaining at that stage of botanical or zoological description and classification, lest they develop a theoretical paradigm and a quantitative modelling approach. Actually, the environmental sciences are less developed in that respect than plate tectonics or planetary sciences, and remain in infancy because too few quantitative concepts have yet been developed, or they are too recent. Such is the case for climate, whereas the ozone problem or that of the ocean cycle is far more advanced.

There are at least two key common points to the geoscientific revolutions: the first is the need for a global approach, the second the importance of time, without which the complex dynamics of the systems cannot be understood. The earth (and the planets) as a whole are the common subject of all studies; earth's behaviour is global, its envelopes are coupled, it has a common history and a single future.

Study of the earth's dynamics and evolution once again required as a basis that time could be measured accurately far back into the past. This has led to the central role of isotope geology, which provides universal methods to study almost all aspects of the earth and planetary sciences. Isotopic tracers are now used in widely different disciplines, such as hydrology, climatology, petrology, geochemistry, cosmochemistry, oceanography, planetology, atmospheric and environmental studies. The same expert can fairly easily switch from one topic to another, still using essentially the same measurement and interpretation techniques. This leads to a great versatility and promotes positive cross-breeding between very different areas (from early meteorites to current ocean circulation, from mantle evolution to river erosion). Isotopes are now a tool, which is central to the earth sciences, in the way the microscope was central to biology in the first part of the 20th century.

Other areas of research, which have an older history and in some cases appeared to be fossilized or only slowly evolving, have been completely renovated by the geoscience revolutions, in which they provided some key observations: such is the case for seismology, magnetism or structural geology. There is, for instance, a parallel to be drawn between magnetism and geochemistry. Although one does not have the broad palette of all chemical elements and their isotopes, the natural magnetism of matter is also a tool that can be used to probe very diverse problems: from the dynamo that operates in the fluid iron part of the earth's core to the magnetic reversals that made it possible for P. Morley, F. Vine and D. Matthews to understand the mechanism of plate drift; from the fossil information that allows us to reconstruct past plate movements to the magnetic sense used by certain species, such as bees, pigeons or turtles, to orient themselves. Combination of geochronology and magnetic reversals has provided the most powerful time-scale, and allowed us to pin down Milankovic climate cycles, to realize the catastrophic scale of continental flood basalts and to understand their possible relationships with continental break-up and episodes of mass extinction of biological species.
The meaning and rhythms of the geological time-scale inherited from our 19th-century predecessors have thus come to be understood.

In the new earth sciences, a fundamental role is played by the science of fluid mechanics. It is through widespread use of its equations and concepts, over an astonishingly broad range of parameters, that one begins to model accurately and to understand better such diverse phenomena as the dynamics of the core in relation to dynamo generation of the magnetic field, convection in the mantle, slab avalanches, plume eruptions and the origin of plate tectonics, dynamics of volcanic eruptions, of oceanic and atmospheric circulation, all the way to formation of oil reservoirs and ore deposits. No modern earth sciences curriculum can do without strong teaching of fluid dynamics.

Much of the earth sciences are of course based on combinations of notions coming from physics and chemistry (and now biology). These cannot be ignored or dissociated. But the earth sciences integrate them and go beyond, in the study of a fascinating and complex object - the earth. Geochemistry is much more than chemistry applied to the earth; the same is true for geophysics and physics. Whoever ignores this performs in an antiquated and now obsolete world. This is largely because the earth must operate within a historical frame and approach. Earth sciences now form the core of modern natural sciences, and provide the unifying paradigm in which one can go through the steps leading from the synthesis of chemical elements to the emergence of the human species, and now its feedback action on the earth itself.

The relevant time-scales are embedded and span over 17 orders of magnitude, from one second to the age of the solar system. Various time-scales are involved and operate in diverse phenomena, and a perspective view is always mandatory. Scaling and coupling, the rich set of tools of nonlinear phenomena come into play. Self-similarity and critical phenomena are at the basis of modern understanding of the cascade leading from a microscopic defect to catastrophic failure of a fault in an earthquake. Some of us believe that there may be more or less strong couplings and causal links between, for instance, fluid flow in the core, plume generation in the deeper mantle, triggering of gigantic volcanic eruptions and massive disruption of climate leading to massive biological extinction events. Large meteorite impacts also have influenced biological evolution, as is the case for the CretaceousTertiary boundary bolide, which appears to have excavated the giant Chicxulub crater in Mexico, 65 Myr ago. Extinction of the dinosaurs might be linked both to this impact and to massive volcanism in the Deccan traps of India. There are indications that volcanic events linked to plume birth may have been important in the last one billion years, but that impacts were far more important in the first 1 Gyr of the earth's history. Unravelling the respective influences of internal and external sources to climate change over geological time is an exciting topic of ongoing research.

Through these various coupling mechanisms, the earth as a whole appears to be a unique integrated system (in the sense of systems theory). How far does this coupling go, how strong are the links and integrations between its 
various components? The rising question is the strength of the link between the two main parts of the natural sciences: geology and biology. The Gaia hypothesis proposes that living species have regulated the climatic regime at the surface of the earth. As often, innovation takes place at the cross-fertilized boundaries between disciplines: biogeochemistry may be a discipline of the future.

As was stated earlier, it has been found that some of the more popular and striking advances in mathematics have a widespread reflection in the earth sciences. Many geo-features are fractal in nature and many of the relevant phenomena are, sometimes strongly, nonlinear. Volcanic eruptions, earthquakes, hurricanes and fingering of water masses in the ocean are all instability phenomena. Land topography, coastal geometry, distribution of tectonic faults and microscopic defects in rocks, cloud shapes, distributions of chemical elements or of biological species all have a fractal component. At the longer time-scales, flood basalts, asteroid impacts or magnetic-field reversals are random events of a catastrophic nature (though the meaning of catastrophe may not be exactly the same). Both fluid dynamics and scaling laws with critical thresholds are providing new ways to unravel the mystery of these magnetic reversals.
Our representation of the earth in the late 1990s, after the three revolutions described in this article are (almost) completed, is drastically different from the one that was still taught until the 1960s. It is far more exciting and dynamic; it is much more comprehensive and wide ranging. It has brought together tens of subdisciplines, many of which previously existed in total isolation. The revolutions may have benefited from 'big science' programmes such as deep drilling or space exploration, but they ultimately were made possible by scores of smart scientists who developed the appropriate observation, measurement and interpretative techniques. Progress often occurred from interactions at the frontiers and boundaries of their disciplines. The palaeontologist started talking to the geochemist, the geologist to the planetary scientist, the volcanologist to the nuclear scientist, the mineralogist to the high-pressure physicist. Now the time has come to widely revise our teaching curricula and to retain the lessons from these exciting decades. We must bring up a new generation of open-minded scientists with broad-ranging interest, training in modelling and field observation, readiness to collaborate across disciplines. This new generation will produce the new ideas without which there is no science. 